- Case Report

\title{
Diabetic Neuropathic Cachexia in a Young Woman
}

\author{
Nurul Azreen Yusof*, Nur Suhaila Idris, Faridah Mohd Zin \\ School of Medical Science, Universiti Sains Malaysia Health Campus, Kubang Kerian, Malaysia
}

Profound weight loss with painful symmetrical peripheral neuropathy in diabetic patients was first described as diabetic neuropathic cachexia more than 4 decades ago. It is a distinct type of diabetic peripheral neuropathy that occurs in the absence of other microvascular and autonomic complications of diabetes. The mechanism and precipitating cause are unknown. It was reported to have good prognosis with spontaneous recovery within months to 2 years. However, it was frequently missed by clinicians because the profound weight loss is the most outstanding complaint, rather than the pain, numbness, or weakness. This often leads to extensive investigation to exclude more sinister causes of weight loss, particularly malignancy. We report a case of a young woman with well-controlled diabetes who presented with profound unintentional weight loss $(26 \mathrm{~kg})$, symmetrical debilitating thigh pain, and clinical signs of peripheral neuropathy. As the disease entity may mimic an inflammatory demyelinating cause of neuropathy, she was treated with a trial of intravenous immunoglobulin, which failed to give any significant benefit. However, she recovered after 6 months without any specific treatment, other than an antidepressant for the neuropathic pain and ongoing rehabilitation.

Keywords: Peripheral Nervous System Diseases; Diabetic Neuropathies; Cachexia; Weight Loss 


\section{INTRODUCTION}

The association between diabetes mellitus (DM) and peripheral neuropathy has been recognized for over 100 years and different subtypes have been described based on the anatomical pattern. The most familiar subtype in clinical practice is diabetic sensorimotor polyneuropathy which typically presents as a slowly-progressive, primarily sensory deficit in a length-dependent fashion, with symptoms starting in the feet and spreading upwards, evoking the classic stocking-glove distribution. ${ }^{1)}$ Some of the rarer associated conditions encountered in clinical practice include diabetic lumbosacral radiculoplexus neuropathy, diabetic amyotrophy, chronic inflammatory demyelinating polyneuropathy (CIDP) in DM, and diabetic neuropathic cachexia (DNC) ${ }^{1)}$ DNC is an extremely rare variant with unknown prevalence. ${ }^{2)}$ It was first described by Ellenberg ${ }^{3)}$ in 1974 based on a group of six patients with mild diabetes who presented with severe weight loss and symmetrical painful peripheral neuropathy. The combination of these two symptoms was so predominant that the admitting diagnosis in each case was either metastatic carcinoma or carcinomatous neuropathy, yet almost all patients recovered spontaneously within a year despite no specific therapy. Because of the cachectic appearance, the term 'diabetic neuropathic cachexia' was suggested. ${ }^{3)}$

Since then, a few additional cases have been reported in the literature. ${ }^{2,45}$ Notably, subsequent case reports described poorly-controlled DM, with symptom resolution when the DM was controlled. ${ }^{4,5)}$ However, the role of aggressive blood glucose control is still not clear. ${ }^{4)}$ It is said that DNC is distinct from other types of diabetic neuropathy as weight loss usually occurs rapidly over a period of 3-6 months and is not related to the severity or duration of DM. ${ }^{6)}$ Autonomic features other than impotence, and microvascular complications such as retinopathy and nephropathy are usually absent in this syndrome. ${ }^{1,6)}$ The pathological basis and ascertainable precipitating cause of DNC have not been determined, ${ }^{2,3,6)}$ but an immune mechanism seems likely. ${ }^{1)}$ The prognosis of DNC is relatively good and spontaneous recovery is expected. ${ }^{3)}$

\section{CASE REPORT}

A 34-year-old woman with well-controlled DM for 1 year presented to a primary care clinic with severe unintentional weight loss of about 26 kg over a 3-month duration, associated with bilateral thigh pain. The symptoms were preceded by a 5-month history of fatigue and anorexia. The pain was confined to the anterior thigh, and was burning in nature with moderate severity (Visual Analog Scale [VAS] score of 5-6). The pain was persistent and more prominent at night, disturbing her sleep. Despite the pain, she was still able to walk, but much slower than before. In addition, she was functionally impaired as she could not go to work or do household chores because of persistent pain and fatigue. She denied weakness, numbness, fever, or any trauma to the thigh. Screening assessments for respiratory, gastrointestinal, hyperthyroid, and connective tissue disease, as well as depression, an eating disorder, and psychosocial stressors, were all negative. Her family his-

Table 1. Test results

\begin{tabular}{|c|c|c|c|}
\hline No. & Date & Test & Results \\
\hline 1 & $23 / 2 / 17$ & Full blood count & Total white cells, 6.21 109/L; hemoglobin, $11.2 \mathrm{~g} / \mathrm{dL} ;$ platelets, 458 10\%/L \\
\hline 2 & 23/2/17 & Renal profile & $\begin{array}{l}\text { Urea, } 4.9 \mathrm{mmo} / \mathrm{L} ; \text { sodium, } 134 \mathrm{mmol} / \mathrm{L} \text {; potassium, } 4.2 \mathrm{mmol} / \mathrm{L} \text {; creatinine, } 91 \mu \mathrm{mol} / \mathrm{L} \text {; } \\
\text { uric acid, } 287 \mu \mathrm{mol} / \mathrm{L}\end{array}$ \\
\hline 3 & 23/2/17 & Liver function test & $\begin{array}{l}\text { Albumin, } 46 \mathrm{~g} / \mathrm{L} \text {; aspartate aminotransferase, } 10 \mathrm{U} / \mathrm{L} \text {; alanine aminotransferase, } 7 \mathrm{U} / \mathrm{L} \text {; } \\
\text { alkaline phosphatase, } 110 \mathrm{U} / \mathrm{L} \text {; total bilirubin, } 10 \mu \mathrm{mol} / \mathrm{L}\end{array}$ \\
\hline 4 & 23/2/17 & Fasting blood sugar & $5.0 \mathrm{mmol} / \mathrm{L}$ \\
\hline 5 & 23/2/17 & Hemoglobin A1C & $6.2 \%$ \\
\hline 6 & $23 / 2 / 17$ & Serum creatine kinase & $29 \mathrm{U} / \mathrm{L}$ \\
\hline 7 & $23 / 2 / 17$ & Erythrocyte sedimentation rate & $36 \mathrm{~mm} / \mathrm{h}$ \\
\hline 8 & 23/2/17 & Thyroid function test & Free T4, $27 \mu \mathrm{g} / \mathrm{dL}$; thyroid stimulating hormone, $0.60 \mathrm{mlU} / \mathrm{L}$ \\
\hline 9 & 23/2/17 & Serum calcium \& phosphate & Serum calcium, $2.25 \mathrm{mmol} / \mathrm{L} ;$ phosphate, $1.55 \mathrm{mmol} / \mathrm{L}$ \\
\hline 10 & $23 / 2 / 17$ & Connective tissue disease screening & $\begin{array}{l}\text { Antinuclear antibody, negative; extractable nuclear antigen, negative; serum C3 and C4 } \\
\text { level, normal; rheumatoid factor, negative }\end{array}$ \\
\hline 11 & $6 / 3 / 17$ & Chest X-ray & 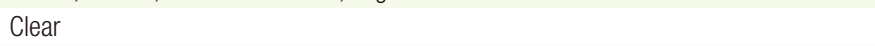 \\
\hline 12 & $6 / 3 / 17$ & Mantoux test & Negative \\
\hline 13 & $6 / 3 / 17$ & Human immunodeficiency virus rapid test & Non-reactive \\
\hline 14 & $6 / 3 / 17$ & Hepatitis B surface antigen & Non-reactive \\
\hline 15 & $6 / 3 / 17$ & Anti-hepatitis C antibody & Non-reactive \\
\hline 16 & $6 / 3 / 17$ & Stool occult blood & Negative \\
\hline 17 & $21 / 3 / 17$ & Ultrasound abdomen & $\begin{array}{l}\text { Multiple small gallstones measuring around } 0.6 \mathrm{~cm} \text {; no sonographic evidence of } \\
\text { malignancy }\end{array}$ \\
\hline 18 & $21 / 3 / 17$ & Bilateral femoral X-ray & Normal bony outline; normal soft tissue shadow \\
\hline 19 & $10 / 4 / 17$ & Urine Bence Jones protein & Negative \\
\hline 20 & $10 / 4 / 17$ & Serum electrophoresis & $\begin{array}{l}\text { Result shows some polyclonal gammopathy which could be due to underlying } \\
\text { inflammatory reactive process. }\end{array}$ \\
\hline
\end{tabular}


tory was noncontributory. She was diagnosed with type 2 DM 1 year prior and was on oral metformin $500 \mathrm{mg}$ and gliclazide $40 \mathrm{mg}$ twice daily since the diagnosis was made. Her baseline hemoglobin Alc (HbAlc) was 6.9\%. No evidence of other diabetic microvascular complications (retinopathy or nephropathy) was found. She took no other medications.

Examination revealed marked wasting and lethargy. There was generalized loss of subcutaneous fat and muscle atrophy. Her previous weight was $68 \mathrm{~kg}$ (body mass index, $25.6 \mathrm{~kg} / \mathrm{m}^{2}$ ) and current weight was $42 \mathrm{~kg}$ (body mass index, $15.5 \mathrm{~kg} / \mathrm{m}^{2}$ ). Her height was $163 \mathrm{~cm}$ and abdominal circumference was $58 \mathrm{~cm}$. Her hydration status was fair. Her random blood sugar was $7.0 \mathrm{mmol} / \mathrm{L}$. There was tenderness on palpation of both quadriceps muscles, but no inflammatory signs were noted. Neurological examination showed muscle wasting of both lower limbs, especially both quadriceps muscles, and hypotonia of both lower limbs with hyporeflexia of both ankle and knee jerks. There was reduced muscle power (grade 4/5) in both quadriceps muscles and proximal myopathy, while the power of other lower limb muscles was graded as $5 / 5$. The sensation in both lower limbs was intact and there was no stocking-glove hypoesthesia.

With consideration of occult malignancy, autoimmune disease, and hyperthyroidism in the differential diagnosis of her weight loss, investigation included basic hematology, biochemistry, autoimmune screening, and inflammatory markers which were all normal. Serum creatine kinase was not increased. Thyroid function tests and tuberculosis workup were normal. Screening for retroviral disease, hepatitis B, and hepatitis $\mathrm{C}$ was negative. Chest and bilateral femoral radiograph, and abdominal ultrasound were unremarkable. The test results are shown in Table 1. The patient also underwent psychiatric assessment, but no definite pathology was found. Repeat HbAlc was $6.2 \%$, while fasting venous blood sugar was $5.0 \mathrm{mmol} / \mathrm{L}$; hence, her oral antidiabetic agents were withheld. She was under primary care follow-up more than a month, causing her to take prolonged medical leave as the pain significantly interfered with her daily life.

In view of the uncertain diagnosis and inconclusive investigations at primary care level, she was admitted to the medical ward for further evaluation. A nerve conduction study (NCS) showed mild slowing of motor conduction velocity in all nerves tested, mildly reduced bilateral peroneal compound muscle action potential, mild conduction block in both peroneal nerves, mild slowing of sensory nerve velocity, and mildly prolonged sensory peak latency in upper limb nerves (Tables 2, 3). The findings were interpreted as possible mild or early onset of patchy demyelinating type of sensorimotor polyneuropathy, probably CIDP. She did not consent to cerebrospinal fluid examination to vali- date the diagnosis of CIDP.

Although the NCS features did not fulfill the diagnostic criteria, she was treated as CIDP based on clinical presentation. She received intravenous immunoglobulin infusion $2 \mathrm{~g} / \mathrm{kg}$ daily for 5 days, in addition to physical and nutritional rehabilitation and ongoing psychological support. She was prescribed a normal balanced diet according to her calculated total daily requirement of $600 \mathrm{kcal} / \mathrm{d}$, with slow increment of $300 \mathrm{kcal} / \mathrm{d}$ every 3 to 4 days. Milk supplementation was added to achieve the caloric intake. The aim of weight gain was $0.5 \mathrm{~kg} / \mathrm{d}$ and she tolerated the refeeding process well. Upon discharge, she was thoroughly instructed in dietary intake to be followed while at home and was seen by the dietician every 4 weeks to monitor her diet and weight gain.

However, during follow-up at neurology clinic 3 weeks after discharge, there was no significant clinical improvement. The thigh pain was persistent and the neurological manifestations were unchanged, with grade 4/5 muscle power in both proximal lower limbs, hyporeflexia of the knee jerk, and areflexia of both ankles. The muscle power in the distal lower limbs was full (grade 5/5). Repeat NCS showed similar findings, indicative of poor response to immunoglobulin. Due to the possibility of association with paraproteinemias that may mimic

Table 3. Motor nerve conduction study (4/4/17)

\begin{tabular}{|c|c|c|c|}
\hline Nerve and site & $\begin{array}{l}\text { Latency } \\
\text { (ms) }\end{array}$ & $\begin{array}{c}\text { Amplitude } \\
\text { (mV) }\end{array}$ & $\begin{array}{c}\text { Conduction } \\
\text { velocity }(\mathrm{m} / \mathrm{s})\end{array}$ \\
\hline \multicolumn{4}{|c|}{ Median nerve (right) } \\
\hline Wrist & 4.5 & 5.6 & \\
\hline Elbow & 9.1 & 5.4 & 45 \\
\hline \multicolumn{4}{|l|}{ Ulnar nerve (right) } \\
\hline Wrist & 4.4 & 8.3 & \\
\hline Below elbow & 8.4 & 7.7 & 45 \\
\hline Above elbow & 10.5 & 7.0 & 52 \\
\hline \multicolumn{4}{|c|}{ Peroneal nerve (right) } \\
\hline Ankle & 5.0 & 1.5 & \\
\hline Fibula & 13.4 & 1.4 & 37 \\
\hline Popliteal fossa & 15.5 & 1.3 & 57 \\
\hline \multicolumn{4}{|l|}{ Peroneal (left) } \\
\hline Ankle & 4.9 & 1.6 & \\
\hline Fibula (head) & 13.7 & 1.4 & 34 \\
\hline Popliteal fossa & 16.0 & 1.5 & 52 \\
\hline \multicolumn{4}{|l|}{ Tibial (right) } \\
\hline Ankle & 5.1 & 7.5 & \\
\hline Popliteal fossa & 14.1 & 5.3 & 44 \\
\hline \multicolumn{4}{|l|}{ Tibial (left) } \\
\hline Ankle & 5.7 & 5.7 & \\
\hline Popliteal fossa & 16.1 & 4.4 & 38 \\
\hline
\end{tabular}

Table 2. Nerve conduction study results (4/4/17)

\begin{tabular}{|c|c|c|c|c|c|c|c|c|c|c|}
\hline \multirow{2}{*}{ Study } & \multicolumn{2}{|c|}{ Median nerve } & \multicolumn{2}{|c|}{ Ulnar nerve } & \multicolumn{2}{|c|}{ Sural nerve } & \multicolumn{2}{|c|}{ Peroneal nerve } & \multicolumn{2}{|c|}{ Tibial nerve } \\
\hline & Right & Left & Right & Left & Right & Left & Right & Left & Right & Left \\
\hline Minimum F wave latency (ms) & 30.2 & - & - & - & - & - & 53.0 & 53.2 & 55.4 & 57.1 \\
\hline Sensory nerve conduction velocity (m/s) & 40 & - & 41 & - & 33 & 33 & 34 & 33 & 33 & 34 \\
\hline
\end{tabular}


CIDP, urine Bence Jones protein and serum paraprotein tests were performed, but were normal. The patient was in great emotional distress and the treating physician was frustrated. Further assessment noted that her symptoms of distress did not fulfil the criteria for depression. Her neuropathy was then treated with daily oral amitriptyline $10 \mathrm{mg}$, with ongoing rehabilitation and psychological support. She was also seen by the dietician and her total daily calorie requirement was increased slowly as tolerated up to 2,500-3,000 kcal/d at 8 weeks after discharge, and was maintained until review at 3-month followup. No complications were noted during the process of nutritional rehabilitation.

During follow-up at 3 months after discharge, she finally achieved a good clinical response after being started on oral amitriptyline in respect to the neuropathic pain, and the VAS score decreased to 2/10. At this point, the diagnosis was revised to DNC in view of the combination of profound weight loss with anorexia, and symmetrical painful paresthesia with evidence of peripheral neuropathy on physical examination. Her appetite returned and her weight was $48 \mathrm{~kg}$ (increased from $42 \mathrm{~kg}$ at first presentation). At 6-month follow-up, she was pain free and the neurological examination was completely normal. During this follow-up, her weight further increased from 48 to $56 \mathrm{~kg}$. She was started back on oral metformin $500 \mathrm{mg}$ twice daily based on latest glucose control, repeat fasting glucose of $6.5 \mathrm{mmol} / \mathrm{L}$ and $\mathrm{HbAlc}$ of $7.0 \%$. The dose remained stable. She returned to work and resumed her normal lifestyle.

Written informed consent was obtained from the patient for publication of this report.

\section{DISCUSSION}

This case illustrates DNC in a young woman. DNC is a rare form of diabetic neuropathy with poorly understood pathogenesis. ${ }^{1)}$ To the best of our knowledge, such a case has not been reported in Malaysia. The initial differential diagnosis of profound weight loss was extensive. Thorough investigation was performed to identify the actual diagnosis, while ruling out more serious pathology, particularly malignancy. The patient presented with symmetrical painful peripheral neuropathy of the bilateral thighs accompanied by profound weight loss of $26 \mathrm{~kg}$ within 3 months and anorexia. At initial presentation, she was thought to have CIDP in DM since all laboratory and radiological investigations were inconclusive. The NCS findings showed some demyelinating features, but did not fulfil the criteria for CIDP. Her condition mimicked CIDP because of the similar subacute to chronic course with painful muscle weakness and areflexia; ${ }^{7)}$ however, weight loss is uncommon in CIDP ${ }^{8)}$ Moreover, CIDP is often associated with insulin-dependent DM as both diseases are thought to have autoimmune etiology, and occasional coexistence might be expected. ${ }^{8)}$

The literature indicates that most other forms of diabetic neuropathy have some demyelinating features on NCS. Therefore, some cases of diabetic peripheral neuropathy may be mislabeled as $\mathrm{CIDP},{ }^{1)}$ as in our patient. In a case report of DNC by Datta et al., ${ }^{5)}$ the patient also had some demyelinating features in the NCS. Typically, in DNC, electromyography and NCS often reveal reduced amplitude of motor and sensory action potentials and mild reduction in conduction velocities, indicating peripheral polyneuropathy from axonal degeneration. ${ }^{6)}$

Another type of diabetic peripheral neuropathy called diabetic amyotrophy, however, closely mimics DNC and could be considered in the differential diagnosis as well. ${ }^{2)}$ Diabetic amyotrophy is also seen in type $2 \mathrm{DM}$, but patients tend to have better glycemic control and lower body mass index. ${ }^{1)}$ Weight loss is also common in this type of neuropathy, with acute to subacute onset of severe lower extremity pain, either in the thigh or the leg. However, the pain in diabetic amyotrophy will ultimately spread to the entire lower extremity. The second difference is asymmetrical involvement in diabetic amyotrophy as opposed to symmetrical presentation in DNC. ${ }^{1)}$ This patient had symmetrical painful neuropathy confined to the anterior thigh. The third difference is that the recovery in diabetic amyotrophy is substantial but incomplete, as most patients often have residual pain and some degree of leg weakness on long-term follow-up. ${ }^{1)}$ In contrast, this patient recovered completely at 6 months after diagnosis and remains well to date. These features further point towards DNC rather than diabetic amyotrophy.

A therapeutic trial of immunoglobulin was given following the diagnosis of possible mild or early onset CIDP. In contrast to the good response in $\mathrm{CIDP}^{7)}$ this patient did not respond well to immunoglobulin therapy. The neuropathic pain and all neurological manifestations were similar after treatment. This made the diagnosis of CIDP less likely, and the diagnosis was revised to DNC. Besides being uncommonly encountered among our diabetic patients, the diagnosis of DNC was not initially considered because diabetic peripheral neuropathy usually occurs in poorly-controlled DM and is rarely associated with marked weight loss in the absence of hyperglycemic manifestations, as illustrated in this case. The presentation of bilaterally symmetrical painful peripheral neuropathy in mild DM associated with profound weight loss and anorexia in the absence of any other identifiable cause of neuropathy support the diagnosis of DNC. ${ }^{1,3)}$

Most cases of DNC have a good prognosis, with a monophasic course and duration of recovery ranging from 12 to 48 months. ${ }^{6}$ DNC can be successfully treated with early and optimal nutritional support, good glycemic control, and pain management. ${ }^{2)}$ In this case, rapid and sustained improvement on a treatment regimen consisting of nutritional intervention, anti-neuropathic medication, and rehabilitation also supported the diagnosis and was consistent with earlier reports of the reversible nature of this condition. She responded well to oral amitriptyline, an antidepressant used in first-line treatment for neuropathic pain, but the doses used are much lower than those for depression. ${ }^{9)}$ Nevertheless, amitriptyline was also effective in treating the depressive symptoms associated with this illness. ${ }^{4}$

Our experience shows that DNC may occur in patients with mild DM. Although DNC is a diagnosis of exclusion, a high index of suspicion in patients with profound weight loss accompanied by debilitating neuropathic pain will lead to timely diagnosis and treatment. This may avoid extensive investigation and unnecessary treatments that are 
not cost-effective. In addition, clinicians need to be aware of this entity as unrecognized cases may lead to severe psychosocial distress, and impaired functioning and quality of life.

\section{CONFLICT OF INTEREST}

No potential conflict of interest relevant to this article was reported.

\section{ORCID}

Nurul Azreen Yusof: https://orcid.org/0000-0002-6418-0659

Nur Suhaila Idris: https://orcid.org/0000-0002-5877-061X

Faridah Mohd Zin: https://orcid.org/0000-0002-7014-4347

\section{REFERENCES}

1. Tracy JA, Dyck PJ. The spectrum of diabetic neuropathies. Phys Med Rehabil Clin N Am 2008;19:1-26.

2. Ugwu ET. Diabetic neuropathic cachexia with profound autonomic dysfunction in a young female with type 1 diabetes. Acta Diabetol 2017;54:1057-60.

3. Ellenberg M. Diabetic neuropathic cachexia. Diabetes 1974;23:418-23.

4. Al-Hajeri T, El-Gebely S, Abdella N. Profound weight loss in a type 2 diabetic patient with diabetic neuropathic cachexia: a case report. Diabetes Metab 2009;35:422-4.

5. Datta S, Arora R, Chitra S, Chakraborty P, Baidya A, Biswas D, et al. Diabetic neuropathic cachexia in a young female. Indian J Endocrinol Metab 2013;17(Suppl 1):S333-4.

6. Godil A, Berriman D, Knapik S, Norman M, Godil F, Firek AF. Diabetic neuropathic cachexia. West J Med 1996;165:382-5.

7. Romedenne P, Mukendi R, Stasse P, Indekeu P, Buysschaert M, Colin IM. An unusual neuropathy in a diabetic patient: evidence for intravenous immunoglobin-induced effective therapy. Diabetes Metab 2001; 27(2 Pt 1):155-8.

8. Krendel DA, Costigan DA, Hopkins LC. Successful treatment of neuropathies in patients with diabetes mellitus. Arch Neurol 1995;52: 1053-61.

9. Benbow SJ, Cossins L, MacFarlane IA. Painful diabetic neuropathy. Diabet Med 1999;16:632-44. 